Article

\title{
Magnetic Field-Induced Reverse Martensitic Transformation and Thermal Transformation Arrest Phenomenon of $\mathrm{Ni}_{41} \mathrm{Cog}_{9} \mathrm{Mn}_{39} \mathrm{Sb}_{11}$ Alloy
}

\section{Rie Y. Umetsu ${ }^{1, *}$, Xiao Xu ${ }^{2}$, Wataru Ito ${ }^{3}$, Takumi Kihara ${ }^{4, \dagger}$, Kohki Takahashi ${ }^{1}$, Masashi Tokunaga ${ }^{4}$ and Ryosuke Kainuma ${ }^{2}$}

1 Institute for Materials Research, Tohoku University, 2-1-1 Katahira, Aoba-ku, Sendai 980-8577, Japan; E-Mail: kohki@imr.tohoku.ac.jp

2 Department of Materials Science, Graduate School of Engineering, Tohoku University, 6-6-02 Aoba, Aramaki-Aza, Aoba-ku, Sendai 980-8579, Japan;

E-Mails: xu@material.tohoku.ac.jp (X.X.); kainuma@material.tohoku.ac.jp (R.K.)

3 Department of Materials and Environmental Engineering, Sendai National College of Technology, 48 Nodayama, Medeshima-Shiote, Natori, Miyagi 981-1239, Japan;

E-Mail: ito@sendai-nct.ac.jp

4 Institute for Solid State Physics, the University of Tokyo, 5-1-5 Kashiwanoha, Kashiwa, Chiba 277-8581, Japan; E-Mails: t_kihara@imr.tohoku.ac.jp (T.K.); tokunaga@issp.u-tokyo.ac.jp (M.T.)

$\dagger$ Current address: Institute for Materials Research, Tohoku University, 2-1-1 Katahira, Aoba-ku, Sendai 980-8577, Japan.

* Author to whom correspondence should be addressed; E-Mail: rieume@imr.tohoku.ac.jp; Tel.: +81-22-215-2470; Fax: +81-22-215-2381.

External Editor: Kurt Ziebeck

Received: 11 November 2014; in revised form: 1 December 2014 / Accepted: 3 December 2014 / Published: 18 December 2014

\footnotetext{
Abstract: In order to investigate behavior of magnetic field-induced reverse martensitic transformation for Ni-Co-Mn-Sb, magnetization experiments up to a static magnetic field of $18 \mathrm{~T}$ and a pulsed magnetic field of $40 \mathrm{~T}$ were carried out. In the thermomagnetization curves for $\mathrm{Ni}_{41} \mathrm{Co}_{9} \mathrm{Mn}_{39} \mathrm{Sb}_{11}$ alloy, the equilibrium transformation temperature $T_{0}$ was observed to decrease with increasing applied magnetic field, $\mu_{0} H$, at a rate of $\mathrm{d} T_{0} / \mathrm{d} \mu_{0} H=4.6 \mathrm{~K} / \mathrm{T}$. The estimated value of entropy change evaluated from the Clausius-Clapeyron relation was about
} 
$14.1 \mathrm{~J} /(\mathrm{K} \cdot \mathrm{kg})$, which was in good agreement with the value obtained by differential scanning calorimetric measurements. For the isothermal magnetization curves, metamagnetic behavior associated with the magnetic field-induced martensitic transformation was observed. The equilibrium magnetic field, $\mu_{0} H_{0}=\left(\mu_{0} H_{\mathrm{Af}}+\mu_{0} H_{\mathrm{Ms}}\right) / 2$, of the martensitic transformation tended to be saturated at lower temperature; that is, transformation arrest phenomenon was confirmed for the Ni-Co-Mn-Sb system, analogous with the Ni(Co)-Mn- $Z$ ( $Z=\mathrm{In}, \mathrm{Sn}, \mathrm{Ga}, \mathrm{Al}$ ) alloys. Temperature dependence of the magnetic field hysteresis, $\mu_{0} H_{\mathrm{hys}}=\mu_{0} H_{\mathrm{Af}}-\mu_{0} H_{\mathrm{Ms}}$, was analyzed based on the model for the plastic deformation introduced by the dislocations. The behavior can be explained by the model and the difference of the sweeping rate of the applied magnetic field was well reflected by the experimental results.

Keywords: Heusler alloy; martensitic transformation; magnetic field-induced transformation; thermal transformation arrest behavior; magnetic structure; magnetic moment

\section{Introduction}

Since a new type of magnetic shape memory alloy was reported for off-stoichiometric NiMn-based Heusler alloys in 2004 [1], related studies have been intensively carried out. This shape memory alloy is different from the conventional one of $\mathrm{Ni}-\mathrm{Mn}-\mathrm{Ga}$, in which a large magnetostriction occurs due to a re-arrangement of the martensitic variant $[2,3]$. In off-stoichiometric Ni-Mn- $Z(Z=\mathrm{In}, \mathrm{Sn}$ and $\mathrm{Sb})$ alloys, a drastic change of magnetization is obtained during the martensitic transformation because the parent phase orders ferromagnetically and the martensite phase shows weak magnetism. Application of a magnetic field decreases the martensitic transformation temperature because of the gain of the Zeeman energy, and thus magnetic field-induced transformation from the martensite phase to the parent phase occurs. This behavior is called the metamagnetic shape memory effect from the experimental fact that almost complete shape recovery was observed during the magnetic field-induced transformation in a Ni-Co-Mn-In specimen in which the strain had been previously introduced [4]. At the same time, as the martensitic transformation, various physical properties also drastically change, such as huge magnetoresistance [5-7], magnetic superelasticity [8], a large inverse magnetocaloric effect [9-12] and large change of the thermal transport properties [13-15]. Therefore, NiMn-based Heusler alloys have attracted interest in both scientific and applicative aspects.

Recently, it has been reported that a thermal transformation arrest phenomenon is observed in Ni-Mn-In [16,17], Ni-Co-Mn-In [5,18], Ni-Co-Mn-Sn [19], Ni-Co-Mn-Ga [20] and Ni-Co-Mn-Al [21-23] alloys, thermally induced martensitic transformation being interrupted below a certain temperature during the magnetic field cooling process, and the parent phase remaining even at the low temperature. The characteristic temperature is called the thermal transformation arrest temperature and is defined as the point where the transformation is interrupted and does not proceed with further cooling. In systematic studies on this phenomenon, it has been found that the equilibrium magnetic field for the martensitic transformation increases with decreasing temperature, and reaches a constant value at lower temperature. This means that the entropy change of the martensitic transformation becomes smaller and smaller with decreasing temperature, and finally becoming zero. That is, it results in a lack of thermal driving force 
of the martensitic transformation. On the other hand, transformation hysteresis in the magnetic field becomes larger at lower temperature. These behaviors are common to the above NiMn-based shape memory alloys showing the thermal transformation arrest phenomenon, and the temperature dependence of the magnetic field hysteresis has been examined by dividing it into two parts, i.e., a thermally activated term and an athermal term [17,18,22,23]. It has been considered that the components of these terms are different in different alloy systems, such as between Ni-Co-Mn-In and Ni-Co-Mn-Al and that the difference would originate in the different energy barriers related to the thermal activations [22,23].

Stoichiometric $\mathrm{Ni}_{2} \mathrm{MnSb}$ with the $L 2{ }_{1}$-type ordered structure is ferromagnetic having a magnetic moment of $4.2 \mu_{\mathrm{B}} / \mathrm{f}$.u. and the Curie temperature of $334 \mathrm{~K}$ [24,25]. In addition, positive pressure dependence of the Curie temperature has been reported by Kaneko et al. [26] and Kanomata et al. [25]. Since the reports of the occurrence of the martensitic transformation in the off-stoichiometric composition [1], a large number of investigations have been performed and many interesting physical properties have been reported in Ni-Mn-Sb and Co-substituted Ni-Co-Mn-Sb alloys, such as large magnetoresistance [27,28], a negative magnetocaloric effect [29,30], a metamagnetic shape memory effect [31] and exchange biasing behavior [32,33], similar to properties of other NiMn-based alloys. In order to investigate metamagnetic behavior and magnetic moment of the field-induced parent phase of $\mathrm{Ni}-\mathrm{Co}-\mathrm{Mn}-\mathrm{Sb}$ alloy, magnetization experiments up to a static magnetic field of $18 \mathrm{~T}$ and a pulsed magnetic field of $40 \mathrm{~T}$ were performed.

\section{Results and Discussion}

\subsection{Metamagnetic Behavior of $\mathrm{Ni}_{41} \mathrm{Co}_{9} \mathrm{Mn}_{39} \mathrm{Sb}_{11}$}

$\mathrm{X}$-ray powder diffraction pattern measured at room temperature for $\mathrm{Ni}_{41} \mathrm{Co}_{9} \mathrm{Mn}_{39} \mathrm{Sb}_{11}$ alloy annealed and quenched from $1173 \mathrm{~K}$ is shown in Figure 1, together with that for a simulated pattern. The alloy is in the parent phase state at room temperature, and the pattern can be indexed as the $L 2{ }_{1}$-type ordered phase. The lattice parameter is refined to be $a=0.5962 \mathrm{~nm}$.

Figure 1. X-ray powder diffraction patterns for $\mathrm{Ni}_{41} \mathrm{Co}_{9} \mathrm{Mn}_{39} \mathrm{Sb}_{11}$ alloy annealed and quenched from $1173 \mathrm{~K}$.

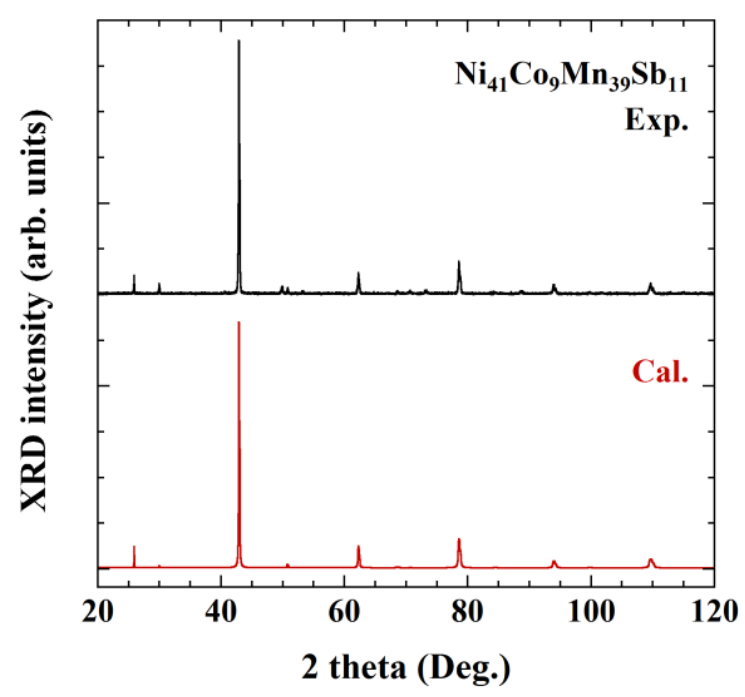


Figure 2a indicates DSC heating and cooling curves measured at a rate of $10 \mathrm{~K} / \mathrm{min}$ for $\mathrm{Ni}_{41} \mathrm{Co}_{9} \mathrm{Mn}_{39} \mathrm{Sb}_{11}$ alloy. $T_{\mathrm{Ms}}, T_{\mathrm{Mf}}, T_{\mathrm{As}}$ and $T_{\mathrm{Af}}$ in the figure are martensitic transformation starting temperature, finishing temperature, and reverse martensitic transformation starting temperature and finishing temperature, respectively. $T_{\mathrm{C}}$ means the Curie temperature and is $368 \mathrm{~K}$. From the DSC measurements, entropy change between the martensite phase and parent phase is obtained to be $15.2 \mathrm{~J} /(\mathrm{K} \cdot \mathrm{kg})$. Thermomagnetization curves measured under applied magnetic fields of $0.05,1,3$ and $5 \mathrm{~T}$ for $\mathrm{Ni}_{41} \mathrm{Co}{ }_{9} \mathrm{Mn}_{39} \mathrm{Sb}_{11}$ alloy are shown in Figure $2 \mathrm{~b}$. It was confirmed that the martensitic transformation temperature decreases with increasing applied magnetic field, and actually decreases by $23 \mathrm{~K}$ when the magnetic field is $5 \mathrm{~T}$. According to the Clausius-Clapeyron relation between magnetism and temperature, the rate of the martensitic transformation temperature change by the magnetic field is given as

$$
\frac{\mathrm{d} \mu_{0} H}{\mathrm{~d} T_{0}}=-\frac{\Delta S}{\Delta M}
$$

where $T_{0}$ is the equilibrium transformation temperature, which is assumed to be $T_{0}=\left(T_{\mathrm{Ms}}+T_{\mathrm{Af}}\right) / 2$. $\Delta M$ is magnetization change between the parent phase and the martensite phase, and $\Delta S$ is the entropy change during the martensitic transformation. From Equation (1), $\Delta S$ is deduced to be $14.1 \mathrm{~J} /(\mathrm{K} \cdot \mathrm{kg})$. This is in good agreement with the value of $15.2 \mathrm{~J} /(\mathrm{K} \cdot \mathrm{kg})$, which is obtained from the DSC measurement in Figure 2a.

Figure 2. (a) DSC heating and cooling curves measured at a rate of $10 \mathrm{~K} / \mathrm{min}$ for $\mathrm{Ni}_{41} \mathrm{Co}_{9} \mathrm{Mn}_{39} \mathrm{Sb}_{11}$ alloy. $T_{\mathrm{Ms}}, T_{\mathrm{Mf}}, T_{\mathrm{As}}$ and $T_{\mathrm{Af}}$ are martensitic transformation starting temperature, finishing temperature, and reverse martensitic transformation starting temperature and finishing temperature, respectively. $T_{\mathrm{C}}$ means the Curie temperature; (b) Thermomagnetization curves were measured under applied magnetic fields of $0.05,1,3$ and $5 \mathrm{~T}$ for $\mathrm{Ni}_{41} \mathrm{Co}_{9} \mathrm{Mn}_{39} \mathrm{Sb}_{11}$ alloy.
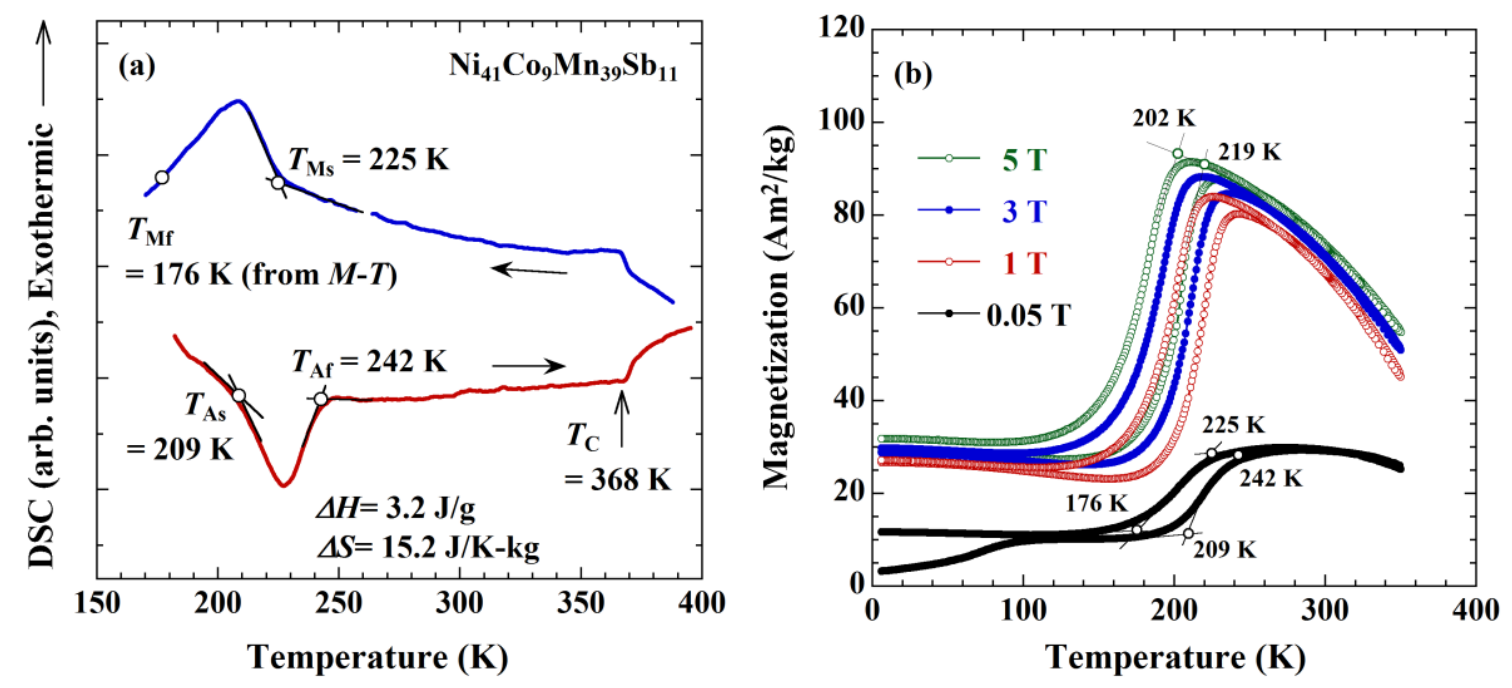

Magnetization curves measured at various temperatures are shown in Figure 3. Here, (a) is that in a static magnetic field up to $18 \mathrm{~T}$ applied by a superconducting magnet; and (b) and (c) are those in a magnetic field up to $40 \mathrm{~T}$ applied by a pulsed magnet. As shown in these figures, metamagnetic behavior attributed to the field-induced reverse martensitic transformation is observed. Here, $\mu_{0} H_{\mathrm{Af}}$ and $\mu_{0} H_{\mathrm{Ms}}$ are 
the critical magnetic fields of the reverse transformation finishing magnetic field and the martensitic transformation starting magnetic field, respectively. It is shown that the critical magnetic fields increase with decreasing measurement temperature and that magnetic field hysteresis becomes enlarged.

Figure 4 is the plot of $\mu_{0} H_{\mathrm{Af}}, \mu_{0} H_{\mathrm{Ms}}$ and the equilibrium martensitic transformation field, $\mu_{0} H_{0}\left(\equiv\left(\mu_{0} H_{\mathrm{Af}}+\mu_{0} H_{\mathrm{Ms}}\right) / 2\right)$, as a function of temperature, together with the reverse martensitic transformation finishing temperature, $T_{\mathrm{Af}}$, martensitic transformation starting temperature, $T_{\mathrm{Ms}}$, and the equilibrium martensitic transformation temperature, $T_{0}\left(\equiv\left(T_{\mathrm{Af}}+T_{\mathrm{Ms}}\right) / 2\right)$, obtained by the DSC and thermomagnetization experiments. $\mu_{0} H_{0}$ and $T_{0}$ are considered to be the equilibrium magnetic field and the equilibrium temperature, respectively, where the Gibbs free energy in the parent phase and that in the martensite phase are equal under the condition that a small amount of martensite phase exists in the matrix of the parent phase [34]. Although $\mu_{0} H_{0}$ increases with decreasing temperature while keeping the same rate, it tends to be saturated under a temperature of about $50 \mathrm{~K}$. The gradient of $\mu_{0} H_{0}$ to temperature is linear to $\Delta S$ as expressed in Equation (1) if $\Delta M$ does not so change. Thus, the approach of $\mathrm{d} \mu_{0} H_{0} / \mathrm{d} T$ to zero means that $\Delta S$ becomes zero. Similar behavior has been observed in Ni-Co-Mn-In [5], Ni-Mn-In [16,17], Ni-Co-Mn-Sn [19], Ni-Co-Mn-Ga [20] and Ni-Co-Mn-Al [21-23]. The temperature at which $\mathrm{d} \mu_{0} H_{0} / \mathrm{d} T$ approaches zero was previously termed kinetically arrest temperature. This is now, however, called "thermal transformation arrest temperature". Basically, the driving force of the thermal transformation, $\Delta G$, can be expressed by $\Delta G \approx \Delta S \cdot \Delta T$, where $\Delta T$ means supercooling. Therefore, the cessation of the transformation when $\Delta S=0$ is caused by the thermodynamic origin and the phenomenon should be named "thermodynamic arrest" [23,35]. The thermal transformation arrest temperature differs for different compositions and/or alloy systems, such as $150 \mathrm{~K}$ in Ni45 $\operatorname{Co}_{5} \mathrm{Mn}_{36.7} \operatorname{In}_{13.3}$ [5], $40 \mathrm{~K}$ in $\mathrm{Ni}_{45} \mathrm{Co}_{5} \mathrm{Mn}_{30} \mathrm{Al}_{20}$ [21], $160 \mathrm{~K}$ in $\mathrm{Ni}_{45} \mathrm{Co}_{15} \mathrm{Mn}_{32.5} \mathrm{Al}_{17.5}$ [23] and $50 \mathrm{~K}$ in the present $\mathrm{Ni}_{41} \mathrm{Co}_{9} \mathrm{Mn}_{39} \mathrm{Sb}_{11}$ alloy. It seems that material having a higher Curie temperature tends to show a higher thermal transformation arrest temperature, however, the specific interrelation between them has not been clarified.

Recently, temperature dependence of the magnetic field hysteresis has been studied for Ni-Mn-In, Ni-Co-Mn-In and Ni-Co-Mn-Al, and a certain level of quantitatively analyses has been successful [17,22,23]. The model is based on the theory of temperature dependence of critical resolved shear stress (CRSS) proposed by Seeger and Kocks et al. [36-38]. Here, the energy of the movement of a dislocation introduced by shear stress is thought to be divided into a thermally activated term and an athermal term. The magnitude of the athermal term is independent of temperature, while the thermally activated term indicates a certain value at $0 \mathrm{~K}$ and decreases with increasing temperature, finally approaching zero at high temperature. Ghosh et al. have found that similar treatment of CRSS is available for the behavior of the martensitic transformation from fcc to bcc as in the concept for temperature dependence of nucleation energy because the diffusionless nature of the martensitic transformation is analogous to plastic deformation [39]. In addition, temperature dependence of stress hysteresis accompanied by stress-induced martensitic transformation for Ti-Ni [40] and Ni-Co-Mn-In [18] has been analyzed by the same model. In the case of Ni-Co-Mn-In, where the transformation can be induced by both magnetic field and stress, temperature dependences of the magnetic field hysteresis and stress hysteresis are similar. Furthermore, dissipation energy, which is required to introduce the stress-induced transformation and magnetic field-induced transformation, has been approximately evaluated and showed same temperature dependences in each other. Therefore, it has been concluded that the effects of the energy applied by the magnetic field and by the stress on the martensitic transformation are 
equivalent [18], suggesting that the application for the model to the phenomenon in the magnetic field-induced martensitic transformation is available.

Figure 3. Magnetization curves measured at various temperatures for $\mathrm{Ni}_{41} \mathrm{Co}_{9} \mathrm{Mn}_{39} \mathrm{Sb}_{11}$ alloy. (a) is measured under static magnetic fields up to $18 \mathrm{~T}$, and (b) and (c) are under pulsed magnetic fields up to $40 \mathrm{~T}$.
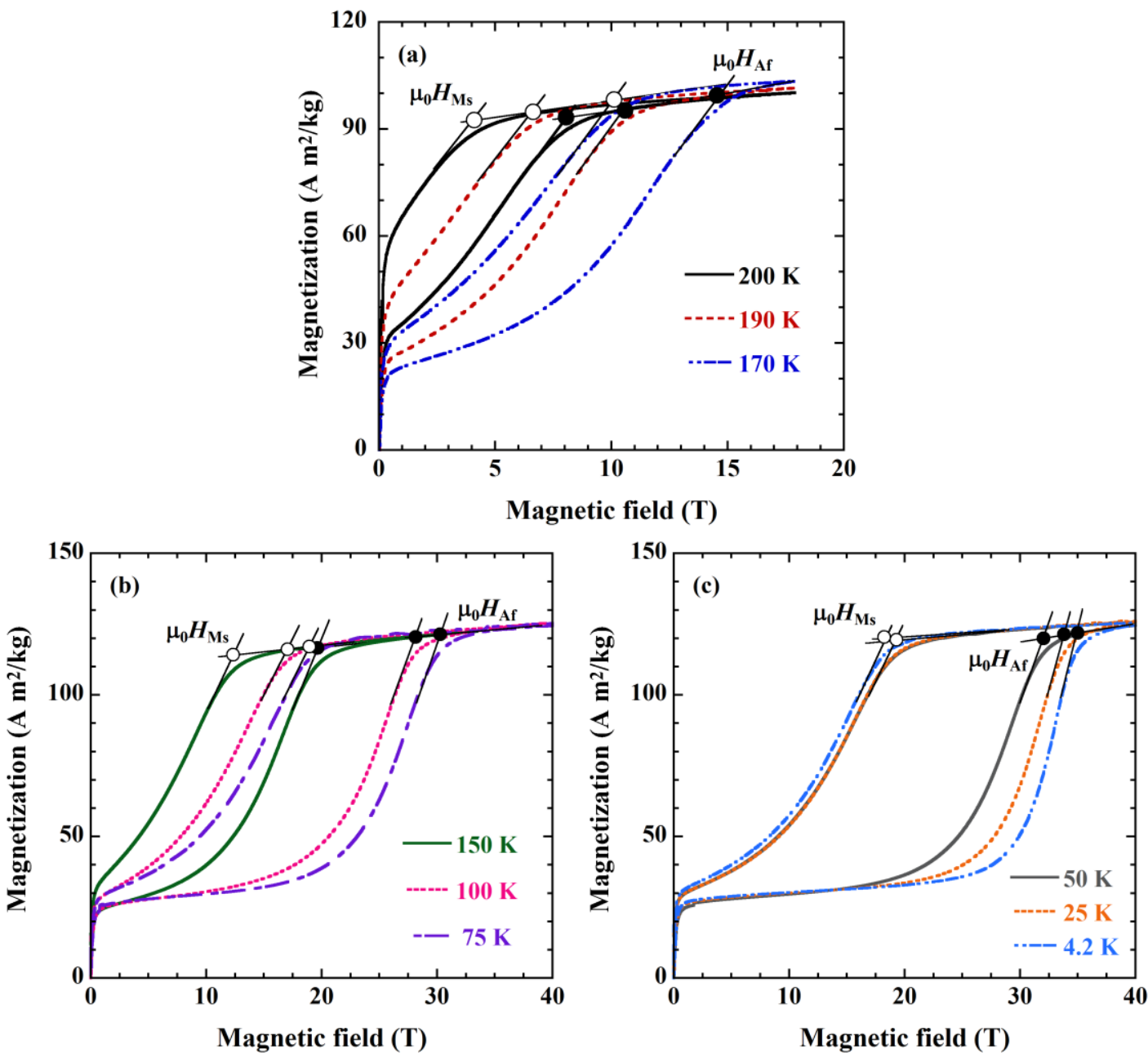

By applying the model to the temperature dependence of the magnetic field hysteresis, Umetsu et al. have proposed the following equation [17]:

$$
\mu_{0} H_{\text {app }}(T)=\mu_{\mathrm{B}} H_{\mu}+\mu_{\mathrm{B}} H_{\mathrm{TA}}(T)=\mu_{\mathrm{B}} H_{\mu}+\mu_{\mathrm{B}} H_{\mathrm{TA}}(0)\left[1-\left(\frac{m k_{\mathrm{B}} T}{Q_{0 \mathrm{~K}}}\right)^{1 / q}\right]^{1 / p}
$$

Here, the first and second terms mean the athermal and thermally activated terms, respectively. $\mu_{0} H_{\mathrm{TA}}(0)$ is the value of $\mu_{0} H_{\mathrm{TA}}(\mathrm{T})$ at $0 \mathrm{~K}$. $Q_{0 \mathrm{~K}}$ is the activation energy necessary to overcome the kinetic barrier without thermal assist and $k_{\mathrm{B}}$ is the Boltzmann constant. $p$ and $q$ are the shape parameters related to the activation barrier. In this phenomenological description, the combination of the $p$ and $q$ is related to the shape of the dislocation-obstacle interaction profile and governs the shape of the $\mu_{0} H_{\text {hys }}-T$ profile. In the case of the magnetic field-induced martensitic transformation, they have been obtained to be $p=1 / 2$, 
$q=3 / 2[17,22,23] . m$ is the kinetic coefficient, and it is written as in the case of the model CRSS by the critical dislocation:

$$
m=\ln \left(\dot{\tau}_{0} / \dot{\tau}\right)
$$

where $\dot{\tau}$ is the rate of the loading speed, $\mathrm{d} \tau / \mathrm{d} t$, and $\dot{\tau}_{0}$ is a characteristic constant for the material. Because the magnetic field-induced martensitic transformation is analogous to stress-induced deformation, Equation (3) can be rewritten as follows:

$$
m=\ln \left(\dot{H}_{0} / \dot{H}\right)
$$

Figure 4. Temperature dependence of the field-induced reverse martensitic transformation finishing field, $\mu_{0} H_{\mathrm{Af}}$, martensitic transformation starting field, $\mu_{0} H_{\mathrm{Ms}}$, and equilibrium martensitic transformation field, $\mu_{0} H_{0}\left(\equiv\left(\mu_{0} H_{\mathrm{Af}}+\mu_{0} H_{\mathrm{Ms}}\right) / 2\right)$, together with the reverse martensitic transformation finishing temperature, $T_{\mathrm{Af}}$, martensitic transformation starting temperature, $T_{\mathrm{Ms}}$, and the equilibrium martensitic transformation temperature $T_{0}\left(\equiv\left(T_{\mathrm{Af}}+T_{\mathrm{Ms}}\right) / 2\right)$ obtained by the DSC and thermomagnetization experiments.

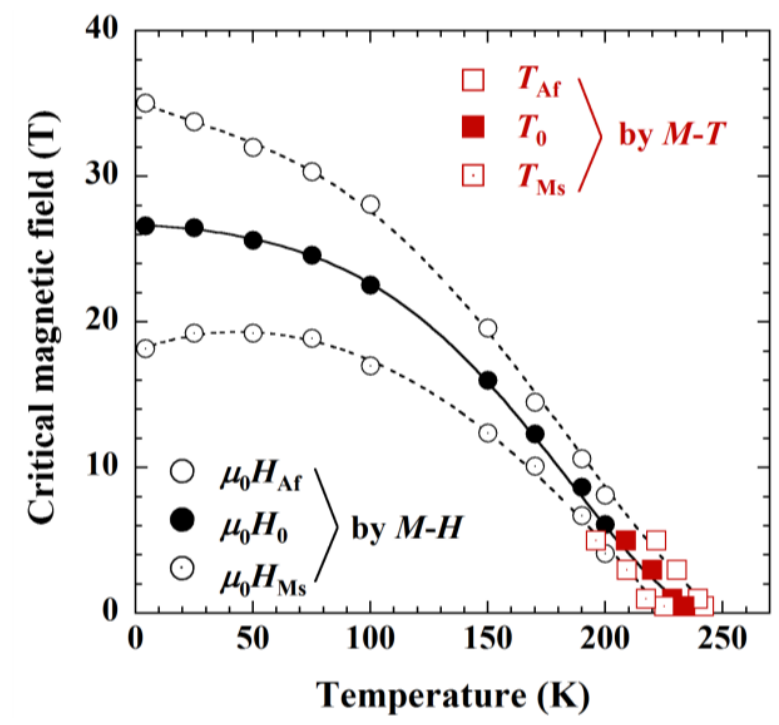

The change of the sweeping rate of the applying magnetic field induces a change of the coefficient of $m$. $\mathrm{Xu}$ et al. demonstrated the systematic changes of the curvature of the temperature dependence of the magnetic field hysteresis by purposely changing the sweeping rate from 0.001 to $10,000 \mathrm{~T} / \mathrm{s}$ for Ni-Co-Mn-In [41]. In the present investigation, the sweeping rate of the magnetic field applied by the superconducting magnet and that applied by the pulsed magnet are about 0.005 and $5000 \mathrm{~T} / \mathrm{s}$, respectively. Temperature dependence of the magnetic field hysteresis, $\mu_{0} H_{\mathrm{hys}}\left(\equiv \mu_{0} H_{\mathrm{Af}}-\mu_{0} H_{\mathrm{Ms}}\right)$, is shown in Figure 5, together with that for NiCoMnIn [5] and NiCoMnAl [22]. Based on Equation (2), fitting of the experimental data was attempted. In the figure, the solid line and dashed line are the fitting curves obtained by Equation (2). The dot-dashed horizontal line stands for the athermal component. Best fitting was obtained when $\mu_{\mathrm{B}} H_{\mu}=3.0(\mathrm{~T})$ and $\mu_{\mathrm{B}} H_{\mathrm{TA}}(0)=15.3(\mathrm{~T})$. Comparing these values with those for Ni-Co-Mn-In and Ni-Co-Mn-Al, it is seen that the value of $\mu_{\mathrm{B}} H_{\mu}$ is between $\mathrm{Ni}-\mathrm{Co}-\mathrm{Mn}-\mathrm{In}$ and Ni-Co-Mn-Al; however, $\mu_{\mathrm{B}} \mathrm{H}_{\mathrm{TA}}(0)$ is comparatively larger than those, indicating that the activation energy of the present $\mathrm{Ni}-\mathrm{Co}-\mathrm{Mn}-\mathrm{Sb}$ is larger than the others. Let us consider activation 
energy more quantitatively. Because the sweeping rate of the applied magnetic field is completely different between that by the superconducting magnet and that by the pulsed magnet, the closed circles and open circles are well fitted individually by the factor of $m k_{\mathrm{B}} / Q_{0 \mathrm{~K}}=0.00198$ and 0.00310 , respectively. From these fitting factors and Equations (2) and (4), $\ln \dot{H}_{0}$ and $Q_{0 \mathrm{~K}}$ can be roughly estimated to be 33.0 and $1.06 \mathrm{eV}$, respectively. $Q_{0 \mathrm{~K}}$ for Ni-Co-Mn-In has been reported to be around $0.7 \mathrm{eV}$ by the same method, which is fitting by the temperature dependence of $\mu_{0} H_{\text {hys }}$ based on the CRSS model [41]. Activation energy of Ni-Mn-Ga for the temperature dependence of the critical stress for twin boundary motion has been reported to be about $0.006-0.04 \mathrm{eV}[42,43]$. On the other hand, the values of $0.6-0.8 \mathrm{eV}$ have also been reported $[43,44]$. This discrepancy would be due to the different structures in the martensite phase or different algorithms used to express the temperature dependence of the characteristic properties [44]. Although further investigations are needed to clarify the mechanism, the present results reflect the difference of the sweeping rate of the applied magnetic field, and the obtained value of the activation energy seems to be reasonable.

Figure 5. Temperature dependence of the magnetic field hysteresis, $\mu_{0} H_{\text {hys }}\left(\equiv \mu_{0} H_{\text {Af }}-\mu_{0} H_{\mathrm{Ms}}\right.$ ), together with that for NiCoMnIn [5] and NiCoMnAl [22]. The solid line and dashed line are the fitting curves obtained by the Equation (2). The dot-dashed horizontal line stands for the athermal component.

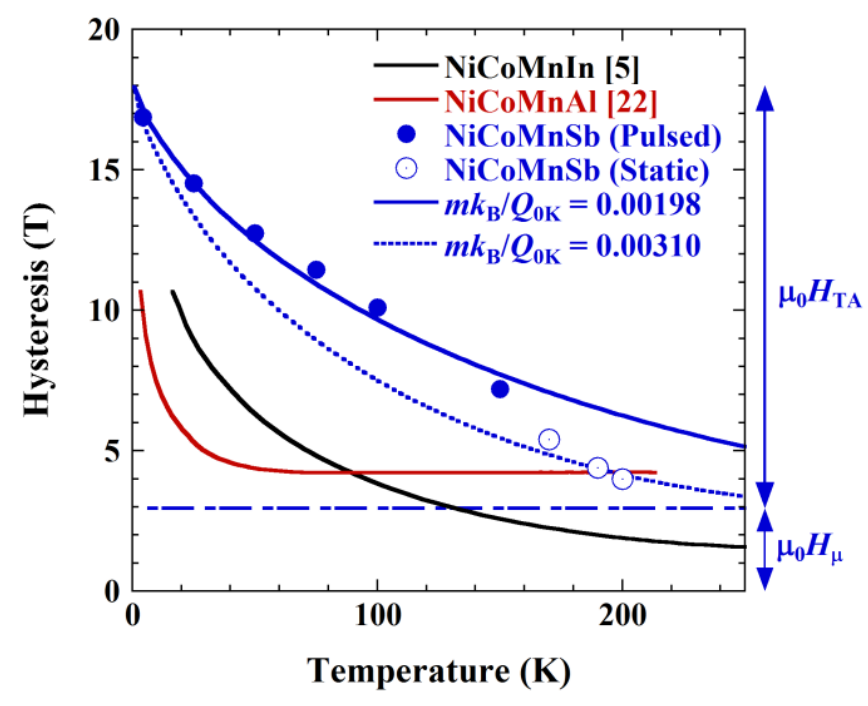

\subsection{Magnetic Moment and Magnetic Structure of $\mathrm{Ni}_{41} \mathrm{Cog}_{9} \mathrm{Mn}_{39} \mathrm{Sb}_{11}$}

Figure $6 \mathrm{a}$ indicates temperature dependence of the spontaneous magnetization, $I_{\mathrm{s}}$, of the present alloy of $\mathrm{Ni}_{41} \mathrm{Co}_{9} \mathrm{Mn}_{39} \mathrm{Sb}_{11}$. Here, $I_{\mathrm{s}}$ is obtained from the Arrott plot $\left(\mu_{0} H / M-M^{2}\right.$ plot) at each measured temperature, as shown in the inset. The curve corresponds to the thermomagnetization curve of the ferromagnetic parent phase. The value of $I_{\mathrm{s}}$ at $4.2 \mathrm{~K}$ is $117.2 \mathrm{~A} \cdot \mathrm{m}^{2} / \mathrm{kg}$, corresponding to the total magnetic moment of $5.4 \mu_{\mathrm{B}} /$ f.u. Concentration dependence of $I_{\mathrm{s}}$ measured at 4.2 or $5 \mathrm{~K}$ for various Ni-based metamagnetic shape memory alloys of $\mathrm{Ni}(\mathrm{Co}) \mathrm{MnIn}, \mathrm{Ni}(\mathrm{Co}) \mathrm{MnSn}$ and $\mathrm{Ni}(\mathrm{Co}) \mathrm{MnSb}$ alloys are shown in Figure 6b $[45,46]$. In the figure, open symbols are $I_{\mathrm{s}}$ for the ternary alloy systems of $\mathrm{Ni}_{50} \mathrm{Mn}_{50-x} \mathrm{In}_{x}(\circ), \mathrm{Ni}_{50} \mathrm{Mn}_{50-x} \mathrm{Sn}_{x}(\square)$ and $\mathrm{Ni}_{50} \mathrm{Mn}_{50-x} \mathrm{Sb}_{x}(\Delta)$, and closed symbols are those for their Co substituted systems. In the case of the $\mathrm{Ni}-\mathrm{Mn}$-In system, $I_{\mathrm{s}}$ increases linearly with a decrease of In content. On the other hand, $I_{\mathrm{s}}$ for Ni-Mn-Sn 
and Ni-Mn-Sb tends to decrease. Especially for Ni-Mn-Sn, it shows an upturn behavior. In the figure, dashed lines of cal.(1) and cal.(2) are expressed from the relations, respectively, as follows:

$$
m_{\mathrm{tot}}=2 m_{\mathrm{Ni}}+m_{\mathrm{Mn}}+\left(1-\frac{x}{25}\right) m_{\mathrm{Mn}}+\frac{x}{25} m_{\mathrm{Sb}}
$$

and

$$
m_{\mathrm{tot}}=2 m_{\mathrm{Ni}}+m_{\mathrm{Mn}}-\left(1-\frac{x}{25}\right) m_{\mathrm{Mn}}+\frac{x}{25} m_{\mathrm{Sb}}
$$

Figure 6. (a) Temperature dependence of the spontaneous magnetization, $I_{\mathrm{s}}$, obtained with the Arrott plot ( $\mu_{0} H / M-M^{2}$ plot) by magnetization curves. Inset is an example of the Arrott plot at $4.2 \mathrm{~K}$; (b) Concentration dependence of $I_{\mathrm{s}}$ measured at 4.2 or $5 \mathrm{~K}$ for various Ni-based metamagnetic shape memory alloys of $\mathrm{Ni}(\mathrm{Co}) \mathrm{MnIn}, \mathrm{Ni}(\mathrm{Co}) \mathrm{MnSn}$ and $\mathrm{NiMnSb}$ alloys $[45,46]$. Open symbols are $I_{\mathrm{s}}$ for the ternary alloy systems of $\mathrm{Ni}_{50} \mathrm{Mn}_{50-x} \mathrm{In}_{x}(\circ), \mathrm{Ni}_{50} \mathrm{Mn}_{50-x} \mathrm{Sn}_{x}(\square)$ and $\mathrm{Ni}_{50} \mathrm{Mn}_{50-x} \mathrm{Sb}_{x}(\Delta)$, and closed symbols are those for their Co substituted systems. Dashed lines of cal.(1) and cal.(2) are expected by Equations (5) and (6), respectively.
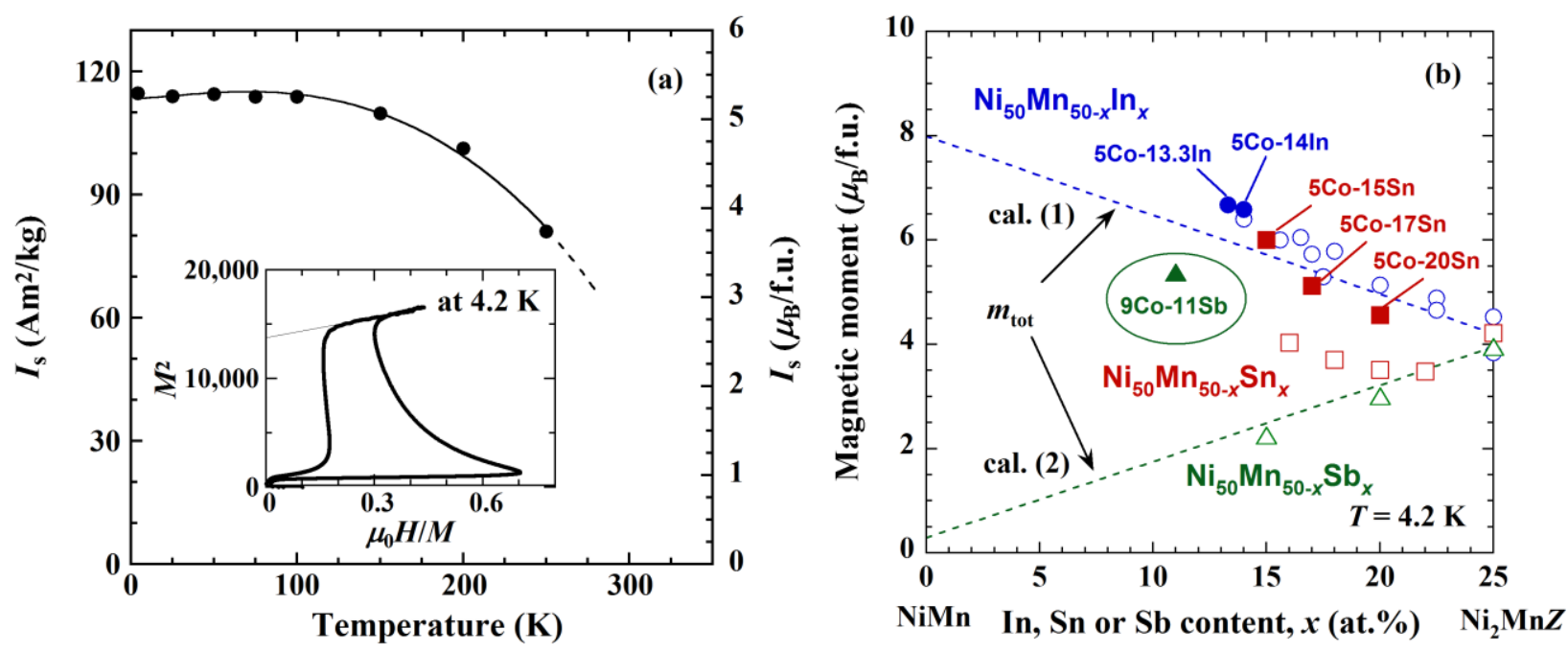

Here, $m_{\mathrm{Ni}}, m_{\mathrm{Mn}}$ and $m_{\mathrm{Sb}}$ are magnetic moments of $\mathrm{Ni}, \mathrm{Mn}$ and $\mathrm{Sb}$ atoms, respectively, and theoretical values of $0.14,3.70$, and $0.14 \mu_{\mathrm{B}}$ for the $\mathrm{Ni}_{2} \mathrm{MnSb}$ stoichiometric alloy are used in the present study [47]. These equations indicate that the slope of the magnetic moment as a function of composition depends on the magnetic configuration of the Mn atoms. That is, line of the cal.(1) is the situation in where the magnetic moment of $\mathrm{Mn}$ at the ordinary ( $4 a$ ) site and at the $\mathrm{Sb}(4 b)$ site couples ferromagnetically; on the other hand, the line of the cal.(2) means it couples antiferromagnetically. Here, it is assumed that the magnetic moment of $\mathrm{Mn}$ at the $\mathrm{Sb}$ site is same as that at the ordinary site and that it only changes the direction of the magnetic moment. Total magnetic moments obtained theoretically for $\mathrm{Ni}_{2} \mathrm{MnIn}$, $\mathrm{Ni}_{2} \mathrm{MnSn}$ and $\mathrm{Ni}_{2} \mathrm{MnSb}$ are 4.21, 4.08 and $3.95 \mu \mathrm{B} /$ f.u., respectively. These values are comparable, and thus the slopes are not thought to be so different in the two systems. Actually, composition dependence of $I_{\mathrm{S}}$ for the Ni-Mn-In system well follows the line of cal.(1) in spite of the magnetic moment of $\mathrm{Ni}_{2} \mathrm{MnSb}$ being used here. This means that the assumption would follow the experimental fact for the Ni-Mn-In system. In addition, for the Ni-Mn-Sb ternary alloy system, behavior of the concentration dependence 
of $I_{\mathrm{s}}$ also well follows the line of cal.(2) indicating antiferromagnetic coupling between the Mn atoms. For Ni-Mn-Sn, the upturn behavior would be due to the change of the magnitude of the magnetic moment and/or the change of the direction of the magnetic moment at the Sn site. Co substituted alloy for the $\mathrm{Ni}-\mathrm{Mn}-\mathrm{Sn}, I_{\mathrm{s}}$ becomes to follow the line of cal.(1), and the change of the magnetic configuration is directly observed by the neutron diffraction experiments [48]. The present result for the Ni-Co-Mn-Sb also indicates evidence of the change of the magnetic configuration, that is, $I_{\mathrm{s}}$ of the present alloy of $\mathrm{Ni}_{41} \mathrm{Co}_{9} \mathrm{Mn}_{39} \mathrm{Sb}_{11}$ becomes larger than that of the total magnetic moment of the stoichiometric composition of $\mathrm{Ni}_{2} \mathrm{MnSb}$. This obtained result is the first evidence of the change of the magnetic configuration for the $\mathrm{Ni}(\mathrm{Co})-\mathrm{Mn}-\mathrm{Sb}$ system as well as the $\mathrm{Ni}(\mathrm{Co})-\mathrm{Mn}-\mathrm{Sn}$ system although the value of $I_{\mathrm{s}}$ for the present alloy does not completely reach the straight line of cal.(1) in Figure $6 \mathrm{~b}$.

\section{Experimental Procedure}

A polycrystalline specimen of $\mathrm{Ni}_{41} \mathrm{Co}_{9} \mathrm{Mn}_{39} \mathrm{Sb}_{11}$ was prepared by induction melting under an argon atmosphere. The obtained ingot was annealed at $1173 \mathrm{~K}$ for 1 day in a vacuum for homogenization, and subsequently quenched into water. An X-ray powder diffraction experiment was conducted for structural identification. Transformation temperatures and entropy change between the martensite phase and parent phase were examined with differential scanning calorimetric (DSC) measurement (Seiko Instruments Inc., Chiba, Japan). Magnetic measurements were performed using the SQUID magnetometer (Quantum Design Japan, Inc., Tokyo, Japan) up to $5 \mathrm{~T}$, by an extraction method up to $18 \mathrm{~T}$ static magnetic fields, and also by an induction method up to $40 \mathrm{~T}$ pulsed magnetic fields. The $18 \mathrm{~T}$ superconducting magnet at the Institute for Materials Research, Tohoku University was employed and a sweeping rate of the applying magnetic field was fixed at $0.005 \mathrm{~T} / \mathrm{s}$. A pulsed magnetic field was obtained with a condenser bank-powered magnet at the Institute for Solid State Physics, The University of Tokyo, its sweeping rate being around $5000 \mathrm{~T} / \mathrm{s}$ in the present experiments.

\section{Conclusions}

In order to study the behavior of the magnetic field-induced reverse transformation of $\mathrm{Ni}_{41} \mathrm{Co}_{9} \mathrm{Mn}_{39} \mathrm{Sb}_{11}$ alloy, magnetic measurements up to a static magnetic field of $18 \mathrm{~T}$ and a pulsed magnetic field of $40 \mathrm{~T}$ were performed.

1. The equilibrium magnetic field, $\mu_{0} H_{0}$, linearly increases with decreasing temperature just below the martensitic transformation temperature. The value of $\mathrm{d} \mu_{0} H_{0} / \mathrm{d} T$ is about $0.22 \mathrm{~T} / \mathrm{K}$ and gives the value of the entropy change during the martensitic transformation $\Delta S=14.1 \mathrm{~J} /(\mathrm{K} \cdot \mathrm{kg})$ from the Clausius-Clapeyron relation. The obtained value is in good agreement with the result given by the DSC measurement of $15.2 \mathrm{~J} /(\mathrm{K} \cdot \mathrm{kg})$.

2. The equilibrium magnetic field, $\mu_{0} H_{0}$, tends to become flat in the low temperature region, showing arresting behavior of the martensitic transformation, analogous with what occurs in the $\mathrm{Ni}(\mathrm{Co})-\mathrm{Mn}-Z(Z=\mathrm{In}, \mathrm{Sn}, \mathrm{Ga}, \mathrm{Al})$ alloys. The thermal transformation arrest temperature of the preset alloy is about $50 \mathrm{~K}$.

3. The temperature dependence of the magnetic field hysteresis, $\mu_{0} H_{\text {hys, }}$, well follows the fitting curves based on the model for the plastic deformation introduced by the dislocations. The difference 
of the sweeping rate of the applied magnetic field is reflected in the experimental results and activation energy of around $1.06 \mathrm{eV}$ for $\mathrm{Ni}_{41} \mathrm{Co}_{9} \mathrm{Mn}_{39} \mathrm{Sb}_{11}$ was obtained.

4. Spontaneous magnetization at $4.2 \mathrm{~K}$ for the magnetically induced parent phase of $\mathrm{Ni}_{41} \mathrm{Co}_{9} \mathrm{Mn}_{39} \mathrm{Sb}_{11}$ is $5.4 \mu \mathrm{B} / \mathrm{f}$.u. This value deviates from the extrapolation from the concentration dependence of the magnetic moment of Ni-Mn-Sb ternary alloy system. The experimental fact suggests a change of the magnetic configuration between $\mathrm{Mn}$ atoms at the $4 a$ site and $\mathrm{Mn}$ at the $4 b$ site by Co substitution.

\section{Acknowledgments}

This study was supported by Grant-in-Aids for Scientific Research (S) and (B), and by the Global COE Program, Tohoku University, from the Japanese Society for the Promotion of Science (JSPS) of the Ministry of Education, Culture, Sports, Science and Technology (MEXT), Japan. This works were performed at High Field Laboratory for Superconducting Materials, Institute for Materials Research, Tohoku University and at the Institute for Solid State Physics, the University of Tokyo. Parts of this work were performed at the Center for Low Temperature Science, Institute for Materials Research, Tohoku University.

\section{Author Contributions}

Rie Y. Umetsu performed the measurements, analyzed the results and wrote the manuscript. Xiao Xu and Wataru Ito carried out the magnetic measurements in high magnetic fields under the collaborations with Takumi Kihara, Kohki Takahashi and Masashi Tokunaga. Ryosuke Kainuma directed the research and conceived the idea for analyzing the results. All authors discussed the results and worked on preparing the manuscript.

\section{Conflicts of Interest}

The authors declare no conflict of interest.

\section{References}

1. Sutou, Y.; Imano, Y.; Koeda, N.; Omori, T.; Kainuma, R.; Ishida, K.; Oikawa, K. Magnetic and martensitic transformations of $\operatorname{NiMn} X(X=\mathrm{In}, \mathrm{Sn}, \mathrm{Sb})$ ferromagnetic shape memory alloys. Appl. Phys. Lett. 2004, 85, 4358-4360.

2. Ullakko, K.; Huang, J.K.; Kanter, C.; Kokorin, V.V.; O’Handley, R.C. Large magnetic-field-induced strains in $\mathrm{Ni}_{2} \mathrm{MnGa}$ single crystals. Appl. Phys. Lett. 1996, 69, 1966-1968.

3. Sozinov, A.; Likhachev, A.A.; Ullakko, K. Crystal structures and magnetic anisotropy properties of Ni-Mn-Ga martensitic phases with giant magnetic-field-induced strain. IEEE Trans. Magn. 2002, $38,2814-2816$.

4. Kainuma, R.; Imano, Y.; Ito, W.; Sutou, Y.; Morito, H.; Okamoto, S.; Kitakami, O.; Oikawa, K.; Fujita, A.; Kanomata, T.; et al. Magnetic-field-induced shape recovery by reverse phase transformation. Nature 2006, 439, 957-960. 
5. Ito, W.; Ito, K.; Umetsu, R.Y.; Kainuma, R.; Koyama, K.; Watanabe, K.; Fujita, T.; Oikawa, K.; Ishida, K.; Kanomata, T. Kinetic arrest of martensitic transformation in the NiCoMnIn metamagnetic shape memory alloy. Appl. Phys. Lett. 2008, 92, 021908.

6. Yu, S.Y.; Liu, Z.H.; Liu, G.D.; Chen, J.L.; Cao, Z.X.; Wu, G.H.; Zhan, B.; Zhang, X.X. Large magnetoresistance in single-crystalline Ni50 $\mathrm{Mn}_{50-x} \mathrm{In}_{x}$ alloys $(x=14-16)$ upon martensitic transformation. Appl. Phys. Lett. 2006, 89, 162503.

7. Sharma, V.K.; Chattopadhyay, M.K.; Shaeb, K.H.B.; Chouhan, A.; Roy, S.B. Large magnetoresistance in Ni50Mn34In 16 alloy. Appl. Phys. Lett. 2006, 89, 222509.

8. Krenke, T.; Duman, E.; Acet, M.; Wassermann, E.F.; Moya, X.; Mañosa, L.; Planes, A. Magnetic superelasticity and inverse magnetocaloric effect in Ni-Mn-In. Phys. Rev. B 2007, 75, 104414.

9. Krenke, T.; Duman, E.; Acet, M.; Wassermann, E.F.; Moya, X.; Manosa, L.; Planes, A. Inverse magnetocaloric effect in ferromagnetic Ni-Mn-Sn alloys. Nat. Mater. 2005, 4, 450-454.

10. Han, Z.D.; Wang, D.H.; Zhang, C.L.; Tang, S.L.; Gu, B.X.; Du, Y.W. Large magnetic entropy changes in the Ni45.4Mnn1.5 $\operatorname{In}_{13.1}$ ferromagnetic shape memory alloy. Appl. Phys. Lett. 2006, 89, 182507.

11. Yu, S.Y.; Ma, L.; Liu, G.D.; Liu, Z.H.; Chen, J.L.; Cao, Z.X.; Wu, G.H.; Zhang, B.; Zhang, X.X. Magnetic field-induced martensitic transformation and large magnetoresistance in NiCoMnSb alloys. Appl. Phys. Lett. 2007, 90, 242501.

12. Liu, J.; Gottschall, T.; Skokov, K.P.; Moore, J.D.; Gutfleisch, O. Giant magnetocaloric effect driven by structural transitions. Nat. Mater. 2012, 11, 620-626.

13. Zhang, B.; Zhang, X.X.; Yu, S.Y.; Chen, J.L.; Cao, Z.X.; Wu, G.H. Giant magnetothermal conductivity in the Ni-Mn-In ferromagnetic shape memory alloys. Appl. Phys. Lett. 2007, 91, 012510 .

14. Koyama, K.; Igarashi, T.; Okada, H.; Watanabe, K.; Kanomata, T.; Kainuma, R.; Ito, W.; Oikawa, K.; Ishida, K. Magnetic and thermoelectric properties of $\mathrm{Ni}_{50} \mathrm{Mn}_{36} \mathrm{Sn}_{14}$ in high-magnetic fields. J. Magn. Magn. Mater. 2007, 310, e994-e995.

15. Sharath, L.S.; Chattopadhyay, C.M.K.; Sharma, V.K.; Roy, S.B.; Pandey, S.K. Temperature dependence of thermoelectric power and thermal conductivity in ferromagnetic shape memory alloy $\mathrm{Ni}_{50} \mathrm{Mn}_{34} \mathrm{In}_{16}$ in magnetic fields. Phys. Rev. B 2010, 81, 195105.

16. Umetsu, R.Y.; Ito, W.; Ito, K.; Koyama, K.; Fujita, A.; Oikawa, K.; Kanomata, T.; Kainuma, R.; Ishida, K. Anomaly in entropy change between parent and martensite phases in the Ni50 $\mathrm{Mn}_{34} \operatorname{In}_{16}$ Heusler alloy. Scr. Mater. 2009, 60, 25-28.

17. Umetsu, R.Y.; Endo, K.; Kondo, A.; Kindo, K.; Ito, W.; Xu, X.; Kanomata, T.; Kainuma, R. Magnetoresistance and transformation hysteresis in the Ni50 Mn34.4In15.6 metamagnetic shape memory alloy. Mater. Trans. 2013, 54, 291-296.

18. Niitsu, K.; Xu, X.; Umetsu, R.Y.; Kainuma, R. Stress-induced transformations at low temperatures in a $\mathrm{Ni}_{45} \mathrm{Co}_{5} \mathrm{Mn}_{36} \mathrm{In}_{14}$ metamagnetic shape memory alloy. Appl. Phys. Lett. 2013, 103, 242406.

19. Umetsu, R.Y.; Ito, K.; Ito, W.; Koyama, K.; Kanomata, T.; Ishida, K.; Kainuma, R. Kinetic arrest behavior in martensitic transformation of NiCoMnSn metamagnetic shape memory alloy. J. Alloy. Compd. 2011, 509, 1389-1393. 
20. Xu, X.; Ito, W.; Umetsu, R.Y.; Koyama, K.; Kainuma, R.; Ishida, K. Kinetic arrest of martensitic

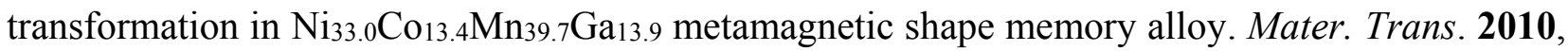
$51,469-471$.

21. Xu, X.; Ito, W.; Tokunaga, M.; Umetsu, R.Y.; Kainuma, R.; Ishida, K. Kinetic arrest of martensitic transformation in NiCoMnAl metamagnetic shape memory alloy. Mater. Trans. 2010, 51, 1357-1360.

22. Xu, X.; Katakura, I.; Kihara, T.; Tokunaga, M.; Ito, W.; Umetsu, R.Y.; Kainuma, R. Optical microscopic study on NiCoMnAl metamagnetic shape memory alloy by in situ observation under a pulsed high magnetic field. Mater. Trans. 2013, 54, 357-362.

23. Xu, X.; Ito, W.; Tokunaga, M.; Kihara, T.; Oka, K.; Umetsu, R.Y.; Kanomata, T. The thermal transformation arrest phenomenon in NiCoMnAl Heusler alloys. Metals 2013, 3, 298-311.

24. Shinohara, T. Nuclear magnetic resonance in Heusler alloys: $\mathrm{Ni}_{2} \mathrm{MnSn}, \mathrm{Co}_{2} \mathrm{MnSn}$ and $\mathrm{Ni}_{2} \mathrm{MnSb}$. J. Phys. Soc. Jpn. 1970, 28, 313-317.

25. Kanomata, T.; Shirakawa, K.; Kaneko, T. Effect of hydrostatic pressure on the Curie temperature of the Heusler alloys $\mathrm{Ni}_{2} \mathrm{MnZ}(Z=\mathrm{Al}, \mathrm{Ga}, \mathrm{In}, \mathrm{Sn}$ and Sb). J. Magn. Magn. Mater. 1987, 65, 76-82.

26. Kaneko, T.; Yoshida, H.; Abe, S.; Kamigaki, K. Pressure effect on the Curie point of the Heusler alloys $\mathrm{Ni}_{2} \mathrm{MnSn}$ and $\mathrm{Ni}_{2} \mathrm{MnSb}$. J. Appl. Phys. 1981, 52, 2046-2048.

27. Nayak, A.K.; Suresh, K.G.; Nigam, A.K. Irreversibility of field-induced magnetostructural transition in NiCoMnSb shape memory alloy revealed by magnetization, transport and heat capacity studies. Appl. Phys. Lett. 2010, 96, 112503.

28. Nayak, A.K.; Suresh, K.G.; Nigam, A.K. Anomalous effects of repeated martensitic transitions on the transport, magnetic and thermal properties in Ni-Co-Mn-Sb Heusler alloy. Acta Mater. 2011, 59, 3304-3312.

29. Nayak, A.K.; Suresh, K.G.; Nigam, A.K.; Coelho, A.A.; Gama, S. Pressure induced magnetic and magnetocaloric properties in NiCoMnSb Heusler alloy. J. Appl. Phys. 2009, 106, 053901.

30. Sahoo, R.; Nayak, A.K.; Suresh, K.G.; Nigam, A.K. Effect of Si and Ga substitutions on the magnetocaloric properties of NiCoMnSb quaternary Heusler alloys. J. Appl. Phys. 2011, 109, 07A921.

31. Du, J.; Zheng, Q.; Ren, W.J.; Feng, W.J.; Liu, X.G.; Zhang, Z.D. Magnetocaloric effect and magnetic-field-induced shape recovery effect at room temperature in ferromagnetic Heusler alloy Ni-Mn-Sb. J. Phys. D Appl. Phys. 2007, 40, 5523-5526.

32. Nayak, A.K.; Suresh, K.G.; Nigam, A.K. Observation of enhanced exchange bias behavior in NiCoMnSb Heusler alloys. J. Phys. D Appl. Phys. 2009, 42, 115004.

33. Khan, M.; Dubenko, I.; Stadler, S.; Ali, N. Exchange bias behavior in Ni-Mn-Sb Heusler alloys. Appl. Phys. Lett. 2007, 91, 072510.

34. Tong, H.C.; Wayman, C.M. Characteristic temperatures and other properties of thermoelastic martensites. Acta Metall. Mater. 1974, 22, 887-896.

35. Cesari, E.; Kustov, S.; Salas, D. Entropy changes in ferromagnetic shape memory alloys. Mater. Sci. Forum 2011, 684, 49-60.

36. Seeger, A. Dislocations and Mechanical Properties; Fisher, J.C., Johnston, W.G., Thomson, R., Vreeland, T., Jr., Eds.; John Wiley: New York, NY, USA, 1957; p. 271. 
37. Kocks, U.F.; Argon, A.S.; Ashby, M.F. Thermodynamics and Kinetics of Slips, Progress in Materials Science; Chalmers, B.J., Christian, W., Massalski, T.B., Eds.; Pergamon Press: Oxford, UK; New York, NY, USA, 1975; Volume 19.

38. Mecking, H.; Kocks, U.F. Kinetics of flow and strain-hardening. Acta Metall. 1981, 29, 1865-1875.

39. Ghosh, G.; Olson, G.B. Kinetics of f.c.c $\rightarrow$ b.c.c heterogeneous martensitic nucleation-II thermal activation. Acta Metall. Mater. 1994, 42, 3371-3379.

40. Niitsu, K.; Omori, T.; Kainuma, R. Stress-induced transformation behaviors at low temperatures in Ti-51.8Ni (at. \%) shape memory alloy. Appl. Phys. Lett. 2013, 102, 231915.

41. Xu, X.; Kihara, T.; Tokunaga, M.; Matsuo, A.; Ito, W.; Umetsu, R.Y.; Kindo, K.; Kainuma, R. Magnetic field hysteresis under various sweeping rates for Ni-Co-Mn-In metamagnetic shape memory alloys. Appl. Phys. Lett. 2013, 103, 122406.

42. Gavriljuk, V.G.; Söderberg, O.; Bliznuk, V.V.; Glavatska, N.I.; Lindroos, V.K. Martensitic transformations and mobility of twin boundaries in $\mathrm{Ni}_{2} \mathrm{MnGa}$ alloys studied by using internal friction. Scr. Mater. 2003, 49, 803-809.

43. Straka, L.; Hänninen, H.; Heczko, O. Temperature dependence of single twin boundary motion in Ni-Mn-Ga martensite. Appl. Phys. Lett. 2011, 98, 141902.

44. Peng, Z.; Jin, X.; Hsu, T.Y.; Pagounis, E. Relaxation of twin boundaries in a $\mathrm{Ni}_{2} \mathrm{MnGa}$ single crystalline. Mater. Sci. Eng. A 2008, 481-482, 310-313.

45. Ito, W.; Xu, X.; Umetsu, R.Y.; Kanomata, T.; Ishida, K.; Kainuma, R. Concentration dependence of magnetic moment in $\mathrm{Ni}_{50-x} \mathrm{Co}_{x} \mathrm{Mn}_{50-y} Z_{y}(Z=\mathrm{In}, \mathrm{Sn})$ Heusler alloys. Appl. Phys. Lett. 2010, 97 , 242512.

46. Miyamoto, T.; Ito, W.; Umetsu, R.Y.; Kainuma, R.; Kanomata, T.; Ishida, K. Phase stability and magnetic properties of $\mathrm{Ni}_{50} \mathrm{Mn}_{50-x} \mathrm{In}_{x}$ Heusler-type alloys. Scr. Mater. 2010, 62, 151-154.

47. Şaşı̆̆lu, E.; Sandratskii, L.M.; Bruno, P. First-principles calculation of the intersublattice exchange interactions and Curie temperatures of the full Heusler alloys $\mathrm{Ni}_{2} \mathrm{Mn} X X=\mathrm{Ga}, \mathrm{In}, \mathrm{Sn}, \mathrm{Sb}$. Phys. Rev. $B$ 2004, 70, 024427.

48. Umetsu, R.Y.; Sheikh, A.; Ito, W.; Ouladdiaf, B.; Ziebeck, K.R.A.; Kanomata, T.; Kainuma, R. The effect of Co substitution on the magnetic properties of the Heusler alloy $\mathrm{Ni}_{50} \mathrm{Mn}_{33} \mathrm{Sn}_{17}$. Appl. Phys. Lett. 2011, 98, 042507.

(C) 2014 by the authors; licensee MDPI, Basel, Switzerland. This article is an open access article distributed under the terms and conditions of the Creative Commons Attribution license (http://creativecommons.org/licenses/by/4.0/). 